\title{
Moral Depravity: Going Beyond Just an Attribute of Psychopathy
}

\author{
Mitchell Rae Spezzaferri ${ }^{\star}$, Gary Collins, Jenny E Aguilar and Anne-Marie Larsen
}

California Baptist University, Riverside, USA

*Corresponding author: Mitchell Rae Spezzaferri, California Baptist University, Riverside, USA, Tel: 19495006376; E-mail: mspezzaferri@calbaptist.edu Received date: May 09, 2017; Accepted date: May 10, 2017; Published date: May 17, 2017

Copyright: (c) 2017 Spezzaferri MR, et al. This is an open-access article distributed under the terms of the Creative Commons Attribution License, which permits unrestricted use, distribution, and reproduction in any medium, provided the original author and source are credited.

\begin{abstract}
Moral depravity is a voluntary violation of morals, values, customs, or manners leading to an aberrant style of personality commonly known as antisocial psychopathy. This study intends to dive deeper, examining moral depravity as more than just an attribute of the psychopathic individual, but rather, the key component to their evolution of psychopathic traits. Through case studies, large group samples, and assessment, the literature has examined the moral upbringing, cognitive reinforcement and relevant theories to investigate how extensively morality affects an individual's emotional and social development. The literature further examines neurobiological correlates between morality and psychopathy thanks to the advancement of neuroimaging technology within the decade. Throughout, researchers and psychologists found specific links between key brain structures of the prefrontal cortex and amygdala to moral emotion processing, moral development, as well as psychopathic traits. Because the correlates between these brain structures overlap, future research, specifically neurobiological research, should investigate how moral depravity affects the etiology of psychopathic traits in antisocial individuals.
\end{abstract}

Keywords Psychopathy; Morality; socialization; Empathy development; Neurobiological bases

\section{Review of the Literature}

\section{Introduction}

Emotions play a large role in human behavior; they are innate and influence many aspects of an individual's socialization and social behavior. Morality is heavily tied to emotions as well as individual and group socialization. So then, morality must have ties to human emotion in order to influence an individual's social behavior. One could also argue that if an individual's moral base is guided by emotions affecting social behavior, then harmful and antisocial behavior, often expressed within psychopathy, can be correlated to an individual's emotional and social development. Pizzaro, et al. [1] found that harmful acts are often informative of moral character, and it is intuitively appealing to think that the magnitude of harm out to directly relate to the moral depravity of an individual. Moral depravity will be used throughout to describe a voluntary lack of values, obligations, customs, and manners eventually leading to poor emotional and social development, oftentimes expressed through psychopathy and other patterns of aberrant behavior. Since few of these topics have been researched and studied in depth, this literature review will attempt to syndicate these variables together in relation to criminal psychopathy, as well as look specifically at the roles morality plays in the emotional and social development of an individual leading to the development criminal psychopathy or psychopathic traits and tendencies.

All humans are born with the capacity for willing, which some elevate as the highest capacity of human beings and all allows human beings to grow as moral agents-to transcend self-interest and open ourselves to others [2]. This capacity for willing is often called human agency or the ability to choose right from wrong, good from evil. Bandura [3]. asserts that the regulation of human conduct invokes much more than just moral reasoning, rather a complex theory of moral agency must link moral knowledge and reasoning to moral conduct. The ability for humans to choose right from wrong is pivotal to our well-being. Strict laws like the ones found in science and mathematics (i.e. Law of Gravitation) do not govern human beings. Instead, due to the complexity of human behavior, humans are governed by moral laws or laws of human nature discerning how we ought to act, but fall short.

These beliefs, desires, and intentions are the critical features of agency, can be informative of moral character, and one's sense of the term "character" is a set of stable intentions to act morally or immorally [1].

From a sociobiological perspective, Williams [4]. found that conflict arises from the individual's freedom or agency and concludes the research stating that morality is the solution to this conflict.

\section{Normative morality}

Shoemaker [5] states that morality is grounded in the brain and that social and moral emotions are very closely tied. By studying different brain regions, chemicals, and neurons, the research asserts that deficits in social behavior are linked to frontal and prefrontal lobe damage resulting in legions. It further asserts that moral emotions arise and develop in an individual later in life, and include guilt, shame, embarrassment, jealousy, pride, and altruism. These emotions function to promote social behavior, a term he coins as normative morality. Green [6] concurs with Shoemaker [5] through thoughts that moral emotions facilitate solutions to social problems; they believe moral and social problems arise from a lack of moral judgment and even states that some morally unappealing emotions, like jealousy, may play indirect roles of promoting social welfare and thus stabilizing moral systems. Both the authors make great claims that morality, emotion, and social behaviors are all closely tied together either through brain physiology and chemistry, or judgments and motivation. Another researcher studying morality and the brain, claims that moral insanity, 
Page 2 of 9

or moral depravity, describes the volition or emotions are diseased without obvious diminution of intellectual capacity [2], and are a natural result of selfishness and individualism often characterized by narcissism. This definition of moral insanity closely resembles and feeds well into what scholars would define as the core of psychopathy today. It is even asserted that morality is socially embedded in human nature through the socialization of emotions $[2,5,6]$.

\section{Functional thinking}

Cushman [6], Green [7] have similar thoughts that moral emotions and functional thinking are very closely tied and state that functional thinking is necessary to understand moral emotions and its purpose is to provide an organizing framework for understanding mechanism and devising new hypotheses, and further asserts that in order to understand moral motivation, researchers must use functional thinking to provide a framework to understand the mechanisms behind moral motivation. Lotze, et al. [8]. see the need for functional thinking as emotional self-regulation in which the individual develops a way to control and manage their emotions. This emotional selfregulation is a process that improves and progresses overtime. In tandem, emotional regulation and moral emotions work together to influence a range of coping capabilities that help enact an individual's social behavior.

\section{Moral emotions}

The moral emotions of guilt and shame help inhibit socially maladaptive behaviors; these are self-relevant emotions that are experienced when someone is doing something morally or social unacceptable [8]. Self-awareness and understanding of standards or rules, the opposite of moral depravity, are necessary for guilt and shame to occur. Guilt is focused on the specific misdeed or failure rather than the self and is the healthier of the two moral emotions. Shame, on the other hand, brings emotional pain along with feelings of worthlessness and powerless leading to negative self-evaluation. Shame generates other emotions such as anger, hostility, sadness, and anxiety. Their research is centered on callous-unemotional traits: arrogance, lack of remorse and empathy, on top of being manipulative and deceitful [8]. These traits lessen one's ability to empathize and they inhibit interpersonal aggression.

\section{Empathy}

Empathy is vital to moral behavior. Without proper emotional and social development, empathy cannot exist. Roskies [9]. researches psychological disorders associated with empathy and impaired moral judgments to answer if empathy necessary for morality. Psychopaths are immoral, and lack of empathy is a key characteristic. Psychopathic behavior can be explained by the fact that empathy is necessary for moral behavior. In the research, calls this the empathy argument for psychopathy and discusses that empathy uses high-level cognitive activities including imitation, altruism, and moral cognition; and empathetic behavior involves facial perception, mirroring appreciation of self and others, as well as having an understanding of social norms [9]. Within psychopathy, the lack of empathetic behavior is due to a deficit in the neural hardware of the brain that when properly functioning regulates empathy and moral cognition.

\section{Abnormal emotional processing}

Blair [10]. conducted research similar to Green [6]. however, associated psychopathy to his studies of moral judgment. Emotion plays a vital role in the moral development of individuals, especially psychopaths-further ties that brain physiology plays a functional role in moral development and therefore psychopathy is built on reinforcement provided by certain emotional experiences $[5,9,10]$. Harenski and Kiehl [11] take it one step further testifying that psychological disorders are characterized by abnormal emotional processing; and this abnormal emotional processing leads to impaired moral judgment. Impairments to moral judgment show abnormalities in the expression of empathy, embarrassment, and guilt, which are diagnostic criterion for antisocial personality disorder [12]. displayed in persons with psychopathic tendencies. These findings guide research in discovering how other psychopathic traits influence emotional processing or vice versa.

\section{Selective moral disengagement}

In the development of the moral self, individuals adopt standards of right and wrong that serve as guides or deterrents from certain conduct [3]. Because individuals have agency, they could choose right from wrong. From a socio-cognitive perspective, they do things that give them a sense of self-satisfaction or self-worth; therefore, morality and moral actions are the product of the interplay between cognitive, affective, and social influences. Bandura further postulates that these moral standards do not function as fixed internal regulators of conduct; instead self-regulatory mechanisms do not operate unless they are activated [3]. The ability to perceive another individual as human activates these moral networks. In the literature, this is often referred to as the Theory of Mind (ToM) and will be discussed further under the neural correlates of psychopathy. Certain humans can disengage these moral networks through reconstructing through moral justifications, dehumanization and minimizing, and describes it as a gradual process that starts off with minor immoral tasks and then escalates as self-censure (moral regulation) is diminished. In other words, they become calloused, unemotional through the emotional, cognitive, and social reinforcement of disengagement. Examples of these disengagements include radical terrorists, Nazis, and even military commanders. The disengagement is conditioned-gradual disengagement of moral self-regulation from violent conduct.

Moral disengagement is already operating in the early years of life; it contributes to social discordance in ways that are likely to lead down dissocial paths [3]. This is often seen in children with an early diagnosis of conduct disorder who are eventually diagnosed with psychopathy, and is believed to be a product of the low guilt they experience over injurious conduct to others. They are less prosocial in their overall development, and moral disengagement against destructive means can be enhanced in children by peer modeling and espousal of peaceable solutions to human conflicts. These destructive means of moral disengagement are social learned and reinforced. Through the lens of Bandura's social-cognitive theory and an interactionist perspective to morality, moral actions are the products of the reciprocal interplay of personal and social influences, and through his research, hypothesizes that moral disengagement is made possible by many of the institutions functioning in our society today, and that to prevent moral disengagement many of the flawed systems and organizations need to drastically change, and therefore, the individual must operate under social safeguards against the misuse of institutional power for exploitative and destructive purposes [3]. In 
other words, it should be more difficult for people to remove humanity and morality from their conduct. It should be more difficult for people to become morally deprived.

Additionally, et al. [13] found links between moral disengagement and aggressive behavior; specifically, that moral disengagements moderates psychopathic traits and aggressive behavior among early adolescents. By examining the relationship between $\mathrm{CU}$ traits, grandiosity, manipulation, impulsivity, and irresponsibility, the researchers found a positive relationship between grandiose/ manipulative traits and instrumental aggression when there were high levels of moral disengagement. More specifically, the moral disengagement process facilitates manipulative individuals' instrumental use of aversive behavior towards other people, and were able associate their findings with psychopathy given that many of the attributes studies are prominent within individuals assessed with psychopathy [13]. Gini and colleagues concluded that, proneness to use moral disengagement mechanisms facilitates different types of aggressive behavior, depending on one's psychopathic traits, and from a developmental perspective, these moral deficits are in place earlier in life and environmental factors, such as parental practices, interact with the individuals personal characterizes placing some children at more of risk of developing further deficits and consequential social maladjustment [13].

As a result, moral disengagement plays a vital role in psychopathy as its key variable in the development of moral and emotional processing possibly leading to less moral activation and additional psychopathic traits.

\section{Psychopathy}

Arvan [14] investigates personality traits and moral judgments to see whether there is a significant positive correlation between antisocial personality traits and moral judgments. His research found that deprived moral judgments eventually developed into antisocial personality traits including machiavellianism, narcissism, and psychopathy. Additionally, socialization through social moral judgment had an impact on the advancement of these traits; however, the criminal or antisocial aspects of these traits, for instance, antisocial psychopathy were not studied [14]. Therefore, this concluded that psychopathy is not always a bad thing if it is prosocial rather than criminal or antisocial in nature, but the same cannot be said for machiavellism and narcissism. Carey holds a similar view by stating that not all violent predators are psychopaths, for one thing, nor are most psychopaths violent criminals $[14,15]$. This emerging field of study on prosocial, or what this literature review will define as successful psychopathy, is beginning to take root as further research is being conducted on individuals with psychopathic traits not relating to criminal deviance. Hare posited that incarcerated psychopaths represent only the tip of a very large iceberg [16].

In their research on the successful development of psychopathy, Lilienfeld, et al. [17]. investigated the individual who displays many of the core features of psychopathic personality while achieving success, and although successful psychopathy is not an oxymoron: it may instead be a variant of psychopathy in which adaptive traits (e.g. superficial charm, social poise) comprising Cleckley [18]. mask are especially prominent. The moderated-expression model would define successful psychopathy as a forme-fruste, or atypical manifestation of psychopathy in which protective factors, such as intact executive functioning, intelligence, and effective parenting buffer psychopathic individuals against maladaptive outcomes [17]. This model supports the theories that moral emotions and motivations, as well as empathy, are socially learned and reinforced $[3,5-\quad 8]$.

\section{Development based on morality and moral disengagement}

Psychopaths are the best examples of the dissociation between knowing well and acting good [19]. This indicates that there may be an inability to regulate behavior despite the availability of requisite knowledge. On the social spectrum, moral behavior stems from a delicate balance between prosocial and altruistic behaviors at one extreme and antisocial and selfish behavior on the other; these behaviors are reinforced in cooperative social interaction: socialization. Through healthy socialization, prosaically and altruistic behaviors become more prevalent among individuals in the groups promoting empathy and the interests of others within the group and/or society. Psychopaths lack this socialization, therefore lacking properly developed empathy and other emotional-social behaviors. Glenn, et al. [20]. explain how this lack of empathy may be related to certain moral domains as well as brain dysfunction.

Socialization should positively reinforce altruistic and prosaically behavior; however, despite the requisite knowledge or reinforced socialization, psychopaths are able to morally disengage to the extent of exercising their criminality. Social cognitive theory adopts an interactionism perspective to morality - moral actions are the product of the reciprocal interplay of cognitive, affective, and social influences, and further noted that moral standards do not function as fixed internal regulators of conduct. These self-regulatory mechanisms do not operate unless they are activated and reinforced [3]. These mechanisms, or controls, are not activated or reinforced when the individual, in this case, the psychopath, is able to disengage from their harmful actions and conduct. Studies indicate that to perceive another as human activated empathetic reactions through perceived similarity [3]. The problem arises; rather disengagement is capable, when the psychopath is unable to activate the empathetic reaction because it has not been emotionally or socially reinforced. It does not exist. Once dehumanized, they are no longer viewed as persons with feelings, hopes, or concerns but as sub-human objects. Moral disengagement is a gradual task and it already operating even in the early years of life by contributing to social discordance in ways that are likely to lead down dissocial paths because psychopaths experience low guilt and remorse over injurious conduct. Oftentimes, this is seen in conduct disorder that eventually manifests into anti-social personality disorder. On the other hand, moral engagement, socially learned and reinforced moral behavior, against destructive means can be enhanced in children by peer modeling and espousal of peaceful solutions to human conflicts. This is also seen in the moderated expression model of successful psychopathy proposed by $[3,17]$.

Gini, et al. [3,13] dove deeper into Bandura's theories on moral disengagement through examining the link between psychopathic traits and aggressive behavior. By describing moral disengagement as a cognitive process whereby moral reasoning (morality) is selectively disengaged from behavior, they found that these mechanisms allow, under certain circumstances, even good people can behave badly [13]. These results confirmed that high levels of moral disengagement increase the strength of the association between grandiose manipulative traits and aggressive behavior. More specifically, the moral disengagement process facilitates a manipulative individual's instrumental use of aversive behavior toward other people. The most important conclusion made is that, regardless of the extent to which 
moral standards are internalized, proneness to the use of moral disengagement facilitates different types of aggressive behavior, depending on one's psychopathic traits... what is presently unknown is the extent to which the observed moral deficits are in place earlier in life and whether early environmental factors (e.g. parental practices) interact with personal characteristics placing some children more at risk of developing such deficits and consequent social maladjustment [13].

Moreover, it is important to recognize from these conclusions that environmental factors, such as social cognition and social reinforcement, play a pivotal role in the ability for a psychopath to morally disengage and therefore violate the rights of others.

\section{Emotional processing and reinforcement}

Psychopathy is primarily associated with impaired harm-based moral reasoning. Harm-based morality stems from dysfunctions of key brain regions associated with response and recognition of facial and vocal cues in addition to reinforcement and appropriate decision making [20]. These regions are linked to emotional and empathic responses to others. Individuals lacking strong feelings of empathy may not find the wellbeing of others to be a salient concern, and thus may not be deterred from engaging in immoral behavior that causes harm to or exploits others. These findings are very similar to research regarding moral judgments and psychopaths. Both studies used and examined the five moral domains and foundations as a basis for moral examination of moral judgments [10]. This research focused on which domain would be most affected by the social and emotional dysfunction commonly linked to psychopathy. Emotional disruptions caused by key brain regions associated with empathy and emotional processing, the brains of psychopaths are unable to emotionally reinforce certain behaviors - particularly behaviors involving the morality or the wellbeing of others $[10,20]$. More on the specific structures is discussed in the following sections.

\section{Biological Bases of Psychopathy and Morality}

James [21] envisaged a three-legged model to plausibly explain the etiology of psychopathy. The three legs are:

1. Unusually low function of the orbital prefrontal cortex and anterior temporal lobe,including the amygdala,

\section{The high-risk variants of several genes, and}

3. Early childhood emotional, social, and physical development. This model is congruent with much of the research being done in the fields of forensic psychology and neuroscience constituting the biological bases for criminal and antisocial behavior. By enacting this model to psychopathy, along with other neuroscience studies, researchers are beginning to better understand, not only how genetics and brain physiology affect individuals' behavior, but also how the individual's social and emotional development affects brain physiology and vice versa.

Given this model [21]. if an individual has the biological predisposition (genetic make-up and brain physiology), the social influences could have a drastic influence on their overall social development and emotional well-being. This includes their ability to connect with other individual's and perceive another individual's experiences as supported by Theory of Mind (ToM) research [21-24].
The past ten years have been a monumental decade in regards to research on the neurology and chemistry of psychological disorders. Advancements in neuroimaging technology have allowed researchers to better understand the biological underpinnings of psychopathy. Thought to be an aberrant style of personality solely influenced by attachment and poor socialization, these recent research findings have laid ground for a more comprehensive understanding of psychopathy, and in particular, how morality plays a role in its biological development. Furthermore, by first understanding the deficits in brain structure and chemistry researchers can begin to comprehend the nature side of the nature v. nurture argument. These biological underpinnings may predispose and individual to moral depravity or psychopathy, but they are not necessarily the defining factors in its development.

Psychopathy and morality are two constructs that are deeply grounded and reinforced in cultural and social behavior. New advancements in research and technology have allowed scientists, psychologists, and psychiatrists to further understand the biological roots pertaining to morality and psychopathy in the brain. The overlap between morality and antisocial disorders is substantial and it is argued that this is partly accounted for by the disruption of the neural systems common to both [23]. Research within the past decade has produced astounding results and discussion on the deficits on the prefrontal cortex and limbic system, as well as the hormones and neurotransmitters most often linked to psychopathy and morality. The remaining literature review will dive into the biological bases of morality as it is applied to the previously known neurological deficits of psychopathy.

\section{Morality}

Morality plays a central role in the constitution of human nature [25]. It integrates its way through cognitive, emotional, and motivational mechanisms of the human brain. From there it is internalized and becomes part of the individual's development enabling social cohesion and organization of behavior, and describe morality as an ethical set of customs and values that are embraced by a cultural group to guide social conduct and behavior. Therefore, any individual(s) that break the cultural moral code are labeled deviant or apart from the norm. In the case of psychopathy, these individuals stray far from the norm in terms of aggression and violence, so much so, that researchers have begun to question if there is more than just a social component to morality. The behaviors can be biologically based in the physiology and hormones of their brain and these predispositions or deficits may be further socialized and reinforced through their interactions and behaviors with other individuals in their cultural communities. By taking a deeper consideration into the biological bases of psychopathy, many answers to those questions begin to emerge.

\section{Psychopathy}

Psychopathy is most accurately described as an aberrant style of personality characterized by a pervasive pattern of disregard for and a violation of the rights of others. These character qualities include, but are not limited to, superficial charm, lack of remorse or empathy, poor judgment and failure to learn from past experiences, unreliability, absence of nervousness, pathological egocentricity and incapacity for love, poverty of affective reaction, loss of insight, impulsive, impersonal, and sexual promiscuity [26]. Though not always deviant, the psychopath is often marked by his or her antisocial personality and 
Page 5 of 9

behavior, but more specifically to this research study, his or her neurological deficits, and brain physiology. Through the development of fMRI technology, researchers have gained a better understanding of how the psychopathic mind works, specifically what areas of the brain are ultimately affecting the individual's abnormal behavior.

Neuroscientists have found evidence that psychopathy scores on the PCL-R reflect physical differences in brain function-Canadian and American researchers reported in a brain-imaging study that psychopaths processed certain abstract words such as grace, future, power differently from non-psychopaths [15]. This could be due to the fact that successful psychopaths may possess higher autonomic responsively, executive functioning, and information processing [17]. It is thought that these variable differences may be protective factors that allow psychopathic traits to be channeled into socially adaptive, or at least less patently antisocial (criminal), manifestations. It is still unclear whether or not these factors are the reason that successful psychopaths are better able to process moral emotions and moral judgments. Further research specific to this population and attribute of psychopathy would be beneficial.

\section{Empathy development and theory of mind}

Connecting with others involves both cold (rational) cognition, where one person understands what others might be thinking and what an appropriate response might be, and hot (emotional/affective) cognition, where one can experience empathy with another's feelings and attitudes-that is, actually feel them much like the other person would experience them [21]. This inability to actually feel what another is experiencing is a key component to psychopathy and moral development, particularly because the incapability for psychopaths to empathize allows them to morally disengage and violate the rights of others. Briefly described, researchers define this empathetic deficit as lacking Theory of Mind (ToM). The same brain regions that are affected by psychopathy are also relevant to the TOM and can be broken down into two levels: lower and upper; the lower level or bottom-down process, involves in the mirror neuron system commonly known as the empathy network [24,27]. This bottom-down process allows for actions mirroring, or emotional contagion, facial emotion recognition, and attention cuing [24]. These processes are implicit and take into account dynamic changes in the environment as well as acknowledge past events and knowledge of the other person. In contrast, the upper level or top-down process refers to the more cognitively demanding ToM processing required for the computation of other people's intention, beliefs, and desires [24].

There is evidence of both affective and cognitive deficits in the component of morality in psychopathic individuals [23]. That is, living an antisocial way of life may change moral thinking to justify the individual's repeated antisocial actions and reduce cognitive dissonance. Furthermore, it has also been argued that psychopaths show excellent (not poor) moral reasoning ability when discussing hypothetical situations; their real failure comes in applying their excellent moral conceptual formulations to guiding their own behavior [18]. These divergent processes further recognize that lower level processing is not enough, and given the increased cognitive demands needed for top-down ToM, it develops later in life and is less automatic and more flexible that the bottom-top processes. In summary, if an individual has the capacity to understand that other people experience different types of mental states, how these mental states arise, how they affect their behavior, and that others may not hold the same mental states in the same environment, then psychopaths generally lack this capacity allowing them to aggress and act violently against others without the empathic responses, both psychologically and physiologically, that an individual without psychopathic tendencies would elicit.

\section{Neural correlates}

First, it is important to recognize that all of the research pertaining to the biological bases of psychopathy is correlations, meaning that even though it is believed that these bases predispose the individuals, they are not causative of their behavior. This is an inevitable limitation to neuroimaging findings, while, in contrast, natural accidents that comprise many of the neurological findings, such as legions or trauma, carry more directly to casualty of psychopathic traits. Over the past ten years, researchers interested in the development of psychopathy have researched specific brain areas that seems to be correlated with psychopathic behavior. The two main areas affected are the prefrontal cortex (PFC) and the limbic system - in particular the amygdala, anterior cingulate cortex, and the insula. Together, the deficits in these areas contribute to the abnormal behavior apparent among individuals with diagnosed psychopathy and those with psychopathic traits because many of these areas of the brain are also associated with morality. The evolution of the moral brain has shaped the functioning of several neural structures, and recent brain imaging studies have begun to explore the neural correlates of moral decision making [28]. Many of these structures appear to be associated with psychopathy. A hypothesis can quickly be made that morality and psychopathy have biological ties that inherently affect a person's behavior.

\section{Prefrontal cortex (PFC)}

The PFC is the executive decision making center of the brain and is most important for the elaboration of personality and character, and the control of impulse, obsession, and antisocial behavior [21]. It is the last area of the brain to develop; full development usually occurs around the age of 25 for males, give or take several years; it is also the first to degrade [29]. Prefrontal deficits have been argued to contribute to the poor decision making, emotional dysregulation, and impaired moral judgments in psychopathic people [30]. and damage to these regions at an early age may lead to even more severe impairments in moral behavior [25]. Raine et al. [31] show that those convicted of predatory murder have relatively good prefrontal functioning whilst those who had committed affective murder have reduced prefrontal activity, which in turn may fail to control emotion regulation. Prefrontal impairments are, perhaps, the best-replicated finding in the imaging literature on antisocial behavior [23]. Knabb and colleagues [29] state that research implicating the role of the PFC in moral decision making has opened new frontiers for understanding legally relevant and interpersonal functions. Furthermore, evolutionary changes to the PFC allowed human morality to merge [32]. The tie between morality and psychopathy is imperative, particularly because given the requisite knowledge of right and wrong, psychopaths are able to morally disengage in order to harm and violate the rights of others. By taking a deeper look into specific brain areas of the PFC, more information on the ability to morally disengage has surfaced.

Orbifrontal Cortex (OFC): One such area of the PFC is the orbifrontal region (OFC). The OFC is part of both the limbic and prefrontal lobes, and is highly involved in emotion, mood, drives, and rewards [33]. it primarily serves to regulate emotion, control mood, monitor rewards and punishments, and it is actively involved in planning and decision-making tasks. In moral decision-making, the 
Page 6 of 9

OFC may be important in integrating moral knowledge with emotional cues as well as disinhibited impulse and behavioral control [23-28]. The OFC demonstrates the brains coordination of the limbic system and PFC processing, especially when it comes to more decision-making. More about the awards based processing will be discussed with the amygdala.

Dorsolateral prefrontal cortex (DLPFC): The dorsolateral prefrontal cortex (DLFPC) is thought to be involved in executive functioning and the ability to organize behavior and solve complex problems, recalling past events, volitional responses to the environment, and planning current actions in a temporally informed manner - this is often associated with cold cognition-concluding that damage to this region does not always results in more aggressive behavior, but DLPFC damage appears to be localized to abnormal executive functioning $[21,29]$. Two fMRI studies have observed abnormal DLPFC functioning in antisocial personality disorder (APD) patients during both an emotional task and in an inhibition task [34,35]. Among psychopaths, the DLPFC was less activated than in healthy individuals, this would suggest that its activity in such a context might only be a byproduct of a process of cognitive control, therefore implicating that it is not involved in the subsystem of moral decision making often reported in the literature [36]. Rather, the ventromedial prefrontal cortex (VMPFC) was actively engaged in those processes. A psychopath has a poorly functioning ventral system, usually used for hot cognition, but may have a normal or even supernormal dorsal system, so that without the bother of conscience and empathy, the cold planning and execution of predatory behaviors become fully tuned, convincingly, highly manipulative, and formidable; because these dorsal systems work so well they can learn how to appear as though they care, thus making them even more dangerous [21].

Ventromedial prefrontal cortex (VMPFC): Blair [37] proposed a neurodevelopmental account of psychopathy in which early-onset dysfunction within ventromedial prefrontal cortex (vmPFC) and amygdala contributes to impaired moral socialization as well as enabling and disabling hot cognition.

The proposal is based on the importance of stimulus-reinforcement associations in moral socialization (learning that certain behaviors are harmful to others and should be avoided) and the role of amygdala and $\mathrm{vmPFC}$ in these processes-the former in valence representation ("good"/“bad"), the latter in outcome expectancy. [38]

Lesions on the VMPFC would therefore induce a dissociation in moral sentiments such as a reduced compassion leading to increased cold-blooded utilitarian, instrumental, or rational, choices during interpersonal dilemmas or increased punishment, anger, and violence towards others [36,39]. Moll et al. [25] found through lesion and functional imaging evidence that damage to the regions of the VMPFC at an early age may lead to even more severe impairments in moral behavior suggesting that moral development can be arrested by early PFC damage. Shoemaker [5] found similar findings in that the VMPFC showed some of the greatest deficits in activity due to diminished connectivity, and presented that there is decreased function between the VMPFC and the amygdala along with other frontal lobe areas. These findings exemplified that it is not just the independent structures that are affecting the behaviors, but in connection and activation with one another the psychopathic brain is severely flawed. Because the VMPFC is involved in representing reinforcement accounts, according to Glenn, et al. [28]. If dysfunctional, the VMPFC may impair appropriate moral decision making. When paired with the reward based functions of the limbic, system, these frontal lobe deficits and dysfunctions become exacerbated when reinforced socially through operant conditioning.

\section{Limbic system}

The limbic system is most commonly referred to as the rewards based center of the brain. This is mainly because the system processes emotion-based stimuli before it is rationally processed by the PFC. The limbic system regulates fear, anger, disgust, trust and happiness; these emotions function to promote survival [5]. These emotions are important because as research the limbic system and its relevance to morality and discovered that limbic circuitry is one of the three key components of social information processing, and further postulate that at the same time the limbic system is developing, the nervous system was co-opted to encode the potential harmful consequences of social separation and distress-the social attachments system borrows the physical neural circuitry, from the limbic system, to encode for social pain [33]. Moll et al. [19]. found similar results stating that the cortico-limbic network is recruited during the performance of moral judgments and decision making. In general, these findings can be summed up in the psychopaths' inability to empathize with others due to the deficits of neural circuitry of the limbic system leading to poor socialization and emotional reinforcement.

Anterior cingulate cortex: The anterior cingulate cortex (ACC) is best known for behavioral motivation. The rostral and central portions of the ACC are associated with the processing of emotion and depression, whereas the dorsal regions have been implicated in cognitive processing and skelemotor activity [29]. In decision-making, conflict detection, rewards tracking, and emotional control [19], and is described as the neural alarm system [33]. It signals when something is wrong or when an automatic process should become effortful. Dysfunction of the ACC results in negative affect, behavior spontaneity alongside motivational and apathetic states. This is perhaps the reason why psychopathic individuals are prone to boredom, not easily aroused, and display a generally flat affect.

Posterior cingulate: Posterior cingulate (PC) is activated during moral dilemmas and serious moral violations [38,40,41]. Among psychopaths, there is a negative association between this activation and their responses to moral dilemmas. These findings may indicate abnormal processing of the PC in psychopaths. More research on this structure is needed to fully understand the effects on psychopathic behavior.

Insula: The insula is an area known to encode disgust and exhibit higher activity when healthy individuals make decisions in difficult personal moral dilemmas [36]. It is also implicated in regulating the negative emotional states of disgust, pain, and hunger. The insula appears to operate as a relay to the limbic system for pain, since the neurocircuitry for empathizing with others is the same as the circuitry for understanding the feelings states of the self, and that the insula and ACC project to and from the amygdala and other limbic structures operating in conjunction with one another [33].

Amygdala: The amygdala receives information from the hypothalamus about peripheral body states like stress and fight or flight-activated in response to emotional and stressful stimuli [33]. Many brain-imaging studies of psychopathy have revealed structural and functional abnormalities in the amygdala [28]. Amygdala hypo responsive to emotional stimuli is a hallmark of the neurobiology of the psychopath [33], reduced activation in the amygdala during the processing of emotional stimuli is one of the most common findings in 
the literature [42-44]. Shirtcliff et al. [33]. stressed that because individuals with hypo responsive amygdalae have difficulty processing their own distress; it is implicated that they will have difficulty processing the distress of others as well. This function of the amygdala extends to how an individual learns to care for others, which would include moral decision-making. Glenn, et al. [28] found that the amygdala is crucial in the formation of stimulus-reinforcement associations, which are important in fear conditioning and may be particularly relevant to socializing children so that they can learn to avoid actions that might harm others, and conclude that without having learned these associations, psychopathic individuals may be undeterred from engaging in acts that benefit themselves at the expense of others.

Blair [45]. similarly state that the amygdala has been implicated in aversive and appetitive conditioning including instrumental learning. The fearful, sad, and happy expressions of emotion all modulate amygdala activity and therefore act as reinforcers for behavior. Glenn et al. [41]. also find that impaired amygdala function appears to disrupt the ability to form stimulus-reinforcement associations, hindering the individual from learning to associate their harmful actions with the pain the distress of others; these deficits further hinder the individual's ability to facilitate empathy for their victims. Glenn et al. [28]. study found similar findings with the amygdala's involvement in learning to associate one's own harmful actions with cues of a victim in distress, psychopaths in particular demonstrate reduced autonomic nervous system responding to cues of distress in others, as well as lacking startle response and ability to identify sad and fearful facial cues and vocal tones. Amygdala regions also impart perception of fear, anger, and disgust in both visual and auditory modalities, leading to deficits in social interactions [19].

Because of its association with the OFC, the amygdala and OFC promote stimulus-reinforcement learning and decision-making also known as operant conditioning. In psychopaths, the hypoactive amygdala fails to trigger a large enough response to emotional stimuli to enhance emotional learning and memory instantiation [33]. This is the main basis for a psychopathic individuals' inability to empathize or make moral decision regarding other people. They are unable to be socially or emotionally reinforced for socially appropriate behavior given their biological make-up or physiological predisposition. On top of neurological abnormalities, chemical differences both influence and reinforce immoral and psychopathic behavior.

\section{Hormones and neurotransmitters}

Cortisol: Shirtcliff et al. [33]. have done extensive research on the effects of cortisol on brain structures; its largest impact appears to be on limbic neurocircuitry and helps explain how emotion-related neurocircuitry can become disrupted for long periods of time or is permanently altered across development. Cortisol is most active in the amygdala and orbifrontal cortex (OFC). Greater amygdala functioning enhances the cortisol stress response and heightened amygdala activity is associated with higher stress cortisol levels; therefore, psychopathic individuals who have reduced amygdala activity show less stress responsivity [33]. In the OFC, the top-down role of cortisol is to index stress activation, and because the PFC generally inhibits limbic activity, it would be assumed that higher PFC, specifically OFC, activity would relate to lower cortisol levels - linking antisocial behavior and psychopathic characteristics to blunted levels and reactivity of cortisol.

Serotonin: Lower amygdala activity also has effects on the neurotransmitter serotonin. In humans, aggressiveness is expressed by feelings of anger, frustration, disgust, and contempt; increased serotonin leads to a decrease in harm avoidance and hostility, and an increase in dominance in social encounters in humans [25]. Preliminary evidence suggests that the narcissism component of psychopathy is related to enhance serotonergic activity [46]. On the other hand, decreased and early depletion activity was found among individuals with psychopathic traits. The findings theorize that these individuals have a disrupted ability to recognize fearful expressions and diminished capacity for performance and emotional reinforcement [47]. Serotonin may also interact with testosterone levels to increase the probability of violent aggression. Evidence suggests that low serotonin levels combined with high testosterone levels augment the rates and intensity of aggression [41].

GABA: The GABA transmitter has an inhibitory or calming effect on the brain and spinal cord; increased levels of GABA are linked with psychopathy [48]. These increase levels of GABA reduce anxiety in psychopaths further decreasing their fear and stress responses as well as their heart rate, respiration, and perfusion during heightened times of arousal in healthy individuals. This hyporeactive state is advantageous to psychopaths because it allows them to think clearly. Their judgment is not clouded by an emotional reaction during strategic moral disengagement and therefore they have no empathetic response and remorse for others. However, it also plays a role in their risk taking and impulsivity. With reduced amounts of the GABA neurotransmitter, this could allow psychopaths to continually feed and reinforce their impulsive cravings because the inhibitory effect GABA has on these impulses would be weaker. Especially when combined with lower activation of the PFC, decision making and impulse inhibition processes would be less effective.

\section{Bio bases conclusion}

The brain is heavily influenced by behavior, but it is also profoundly influential on behavior. The physiology and neurochemistry of psychopaths is something to be studied. The combination of limbic system impairment and prefrontal cortex dysfunction create a cluster of emotional, cognitive, and motivational deficits that are linked to severe social impairment due to their lack of morality, remorse, and ability to empathize with others. Research regarding the biological bases of morality and psychopathy has come a long way in the past ten years due to increases in research funding as well as advancements in brain imaging technology, but scientists still have a long way to go. Glenn and Raine [41] summarize it best,

It is becoming increasingly clear that understanding the neurobiology of psychology goes far beyond identifying brain regions that may be involved. Genetics, neurotransmitters, and hormones all impact the functioning of brain structures and connectivity between them.

Additionally, the more researchers, psychologists, and therapists understand about the biological underpinnings of psychopathy rooted in the dysfunction of moral decision making, the better able they will be at assessing and treating the aberrant style of personality known as psychopathy.

\section{Conclusion}

Altogether, there is quite a bit of material regarding how emotion and morality are tied into social behavior and the development of psychopathy, and this literature review focuses on how these variables are linked to negative social behavior and antisocial psychopathy. 
Page 8 of 9

Much of the current literate discusses the moral judgments, depravity, and disengagement of psychopathic individuals and how they affect their social behavior in a negative or positive manner. Brain physiology was also generously examined, and this topic can be useful in the study of how moral depravity affects emotional brain development within the social brain. Deficits to certain brain structures may have an overwhelming effect on individual social, emotion, and overall moral development. All in all, the current literature gives insight into morality, emotion, socialization, and psychopathy as individual variables that do not have strong or significant correlations. In future literature, the relationship between moral depravity, negative emotional affect, and inadequate socialization leading to poor social behavior would be studied in its association to psychopathy or psychopathic deviance.

\section{References}

1. Pizzaro DA, Tannenbaum D, Uhlmann E (2012) Mindless, harmless, and blameworthy. Psychol Inq 2: 185-188.

2. Boyer J (2014) Religion, moral insanity, and psychology in nineteenthcentury America. J Interpret Res 24: 70-99.

3. Bandura A (2002) Selective moral disengagement in the exercise of moral agency. J Moral Educ 31: 102-116.

4. Williams PA (2000) Sociobiology of original sin. Zygon 35: 783-811.

5. Shoemaker WJ (2012) Doing good, doing bad, doing nothing. Zygon 47: 806-817.

6. Green JD (2011) Emotion and morality: A tasting menu. Emot Rev 227-228.

7. Cushman F (2011) Moral Emotions from the Frog's Eye View. Emot Rev 3: 261-263.

8. Lotze GM, Ravindran N, Myers BJ (2010) Moral emotions, emotional self-regulation, callous-unemotional traits, and problem behavior in children of incarcerated mother. J Child Fam Stud 19: 702-713.

9. Roskies A (2011) A Puzzle About Empathy. Emot Rev 3: 278-280.

10. Blair R (2011) Moral Judgment and Psychopathy. Emot Rev 296-298.

11. Harenski CL, Kiehl KA (2011) Emotion and morality in psychopathy and paraphilias. Emot Rev 3: 299-303.

12. American Psychiatric Association (2013) Diagnostic and statistical manual of mental disorders (5th ed.) Washington, DC.

13. Gini G, Pozzoli T, Bussey K (2015) Moral disengagement moderates the link between psychopathic traits and aggressive behavior among early adolescents. Merrill-Palmer Quarterly 61: 51-67.

14. Arvan M (2013) Bad new for conservatives? Moral judgments and the dark triad personality traits: A correlation study. Neuroethics 6: 307-318.

15. Carey B (2008) For the worst of us, the diagnosis may be 'evil'. New York Times.

16. Hare RD (1993) Without conscience: The disturbing world of the psychopaths among us. New York NY: The Guilford Press.

17. Lilienfel SO, Watts AL, Smith SF (2015) Successful psychopathy: A scientific status report. Sage Publications 24: 298-303.

18. Cleckley HM (1941) The mask of sanity: An attempt to clarify some issues about so-called psychopathic personality. Maryland Heights MO: CV Mosby Co.

19. Moll J, de Oliveira-Souza R, Eslinger PJ (2003) Morals and the human brain: A working model. Neuroreport 14: 299-305.

20. Glenn AL, Iyer R, Graham J, Koleva S, Haidt J (2009) Are all types of morality compromised in psychopathy? J Pers Disord 23: 384-398.

21. Fallon J (2013) The Psychopath Inside. New York NY: Penguin Group.

22. Blair RJ, Peschardt KS, Budhani S, Mitchell DG, Pine DS (2006) The development of psychopathy. J. Child Psychol. Psychiatry 47: 262-276.

23. Raine A, Yang Y (2006) Neural foundations to moral reasoning and antisocial behavior. Soc Cogn Affect Neurosci 1: 203-213.
24. Sharp C, Vanwoerden S (2014) The developmental building blocks of psychopathic traits: Revisiting the role of theory of mind. J. Pers. Disord 28: 78-95.

25. Moll J, De Oliveira-Souza R, Zahn R (2008) The neural basis of moral cognition: Sentiments, concepts, and values. Ann N Y Acad Sci 1124: 161-180.

26. Meloy R (2015) Assessing psychopathy with the psychopathy checklist revised (PCL-R).

27. Hay DF (2014) Social cognition: Commentary: Do theory of mind deficits lead to psychopathology or is it the other way around? J Pers Disord 28: 96-100.

28. Glenn AL, Raine A, Schug RA (2009) The neural correlates of moral decision-making in psychopathy. Mol. Psychiatry 1: 5-6.

29. Knabb JJ, Welsh RK, Ziebell JG, Reimer KS (2009) Neuroscience, moral reasoning, and the law. Behav Sci Law 27: 219-236.

30. Gao Y, Glenn AL, Schug RA, Yang Y, Raine A (2009) The neurobiology of psychopathy: A neurodevelopmental perspective. Can J Psychiatry 54: 813-823.

31. Raine A, Meloy JR, Bihrle S, Stoddard J, LaCasse L, et al. (1998) Reduced prefrontal and increased subcortical brain functioning assessed using positron emission tomography in predatory and affective murderers. Behav Sci Law, 16: 319-332.

32. Moll J, Zahn R, de Oliveira-Souza R, Krueger F, Grafman J (2005) Opinion: The neural basis of human moral cognition. Nat Rev Neurosci 6: 799-809.

33. Shirtcliff EA, Vitacco MJ, Graf AR, Gostisha AJ, Merz JL, et al. (2009) Neurobiology of empathy and callousness: Implications for the development of antisocial behavior. Behav Sci Law 27: 137-171.

34. Schneider F, Habel U, Kessler C, Posse S, Grodd W, et al. (2000) Functional imaging of conditioned aversive emotional responses in antisocial personality disorder. Neuropsychobiology: 192-201.

35. Vollm B, Richardson P, Stirling J (2004) Neurobiological substrates of antisocial and borderline personality disorders: Preliminary result of a functional fMRI studyCrim Behav Ment Health 1: 39-54.

36. Tassy S, Oullier O, Cermolacce M, Wicker B (2009) Do psychopathic patients use their DLPFC when making decisions in moral dilemmas? Mol. Psychiatry 14: 908-909.

37. Blair RJ (2007) The amygdala and ventromedial prefrontal cortex in morality and psychopathy. Trends Cogn Sci 11: 387-392.

38. Harenski CL, Edwards BG, Harenski KA, Kiehl KA (2014) Neural processing of moral violations among incarcerated adolscents with psychopathic traits. Dev Cogn Neurosci 10: 181-191.

39. Snowden RJ, Gray N, Smith J, Morris M, Macculloch MJ (2004) Implicit affective associations to violence in psychopathic murderers. J Forens Psychiatry Psychol 15: 620-641.

40. Harenski CL, Hamann S (2006) Neural correlates of regulating negative emotions related to moral violations. Neuroimage 30: 313-324.

41. Glenn AL, Raine A (2008) The neurobiology of psychopathy. Psychiatr Clin North Am 31: 463-47, vii.

42. Kiehl KA, Smith AM, Hare RD, Mendrek A, Forster BB, et al. (2001) Limbic abnormalities in affective processing by criminal psychopaths as revealed by functional magnetic resonance imaging. Biol. Psychiatry 50: 677-684.

43. Veit R, Flor H, Erb M (2002) Brain circuits involved in emotional learning in antisocial behavior and social phobia in humans. Neurosci. Lett. 328 233-236.

44. Birbaumer N, Veit R, Lotze M, Erb M, Hermann C, et al. (2005) Deficient fear conditioning in psychopathy: A functional magnetic resonance imaging study. Arch Gen Psychiatry 62: 799-805.

45. Blair RJ, Mitchell DG (2009) Psychopathy, attention and emotion. Psychol Med 39: 543-555.

46. Stellwagen KK, Kerig PK (2013) Ringleader bullying: Association of with psychopathic narcissism and theory of mind among child psychiatric patients. Child Psychiatry Hum Dev 4: 612-620. 
Citation: Spezzaferri MR, Collins G, Aguilar JE, Larsen AM (2017) Moral Depravity: Going Beyond Just an Attribute of Psychopathy. J Foren Psy 2: 122. doi:10.4172/2475-319X.1000122

Page 9 of 9

47. Blair RJ (2013) The neurobiology of psychopathic traits in youths. Nat Rev Neurosci 14: 786-799.
48. Carlson NL (2010) Physiology of behavior (10th ed.) Boston MA: Pearson Higher Education. 